Supporting Information for

\title{
Enantioselective interaction between cells and chiral hydroxyapatite films
}

Chao Zhou ${ }^{1 \dagger}$, Shaoyang Zhang ${ }^{2 \dagger}$, Jing $\mathrm{Ai}^{3 \dagger}$, Ping $\mathrm{Li}^{2}$, Yanjie Zhao ${ }^{1}$, Baojie $\mathrm{Li}^{2 *}$, Lu Han ${ }^{3 *}$, Yingying Duan $^{3 *}$, Shunai Che ${ }^{1,3^{*}}$

${ }^{1}$ School of Chemistry and Chemical Engineering, State Key Laboratory of Composite Materials, Frontiers Science Center for Transformative Molecules, Shanghai Key Laboratory for Molecular Engineering of Chiral Drugs, Shanghai Jiao Tong University, 800 Dongchuan Road, Shanghai, 200240, China.

${ }^{2}$ Bio-X Institutes, Key Laboratory for the Genetics of Developmental and Neuropsychiatric Disorders, Shanghai Jiao Tong University, Ministry of Education, 800 Dongchuan Road, Shanghai, China;

${ }^{3}$ School of Chemical Science and Engineering, Tongji University, 1239 Siping Road, Shanghai, 200092, China.

these authors contributed equally to this work.

*Correspondence to: chesa@sjtu.edu.cn; libj@sjtu.edu.cn; luhan@tongji.edu.cn; yyduan@tongji.edu.cn 
Supporting Information contains:

Supplementary Figures $\quad$ S1 to 20

Captions for movies S1 to S4

Other supplementary material for this manuscript includes:

Movies S1 to S4

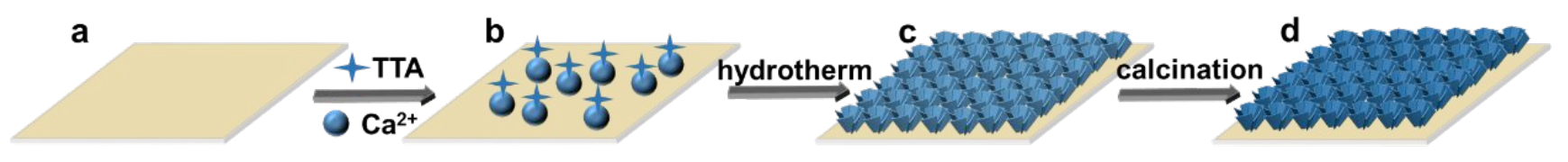

Fig. S1 | Fabrication of CMHAPFs. a, mica wafer was cleaned with ethanol and acetone and then use transparent tape to peel the purchased mica to obtain a fresh surface. $\mathbf{b}$, Calcium phosphate seeds were grown on the mica substrate in a homogeneous solution composed of tartaric acid, calcium salt, phosphate, urea and deionized water under $180{ }^{\circ} \mathrm{C}$. c, Formation of chiral mesostructured hydroxyapatite films. d, Pure inorganic chiral mesostructured hydroxyapatite films (CMHAPFs) was obtained after calcination. The mesostructures of CMHAPFs could be controlled by adjusting the concentrations of tartaric acid, reaction time and $\mathrm{pH}$ of the reaction system. 


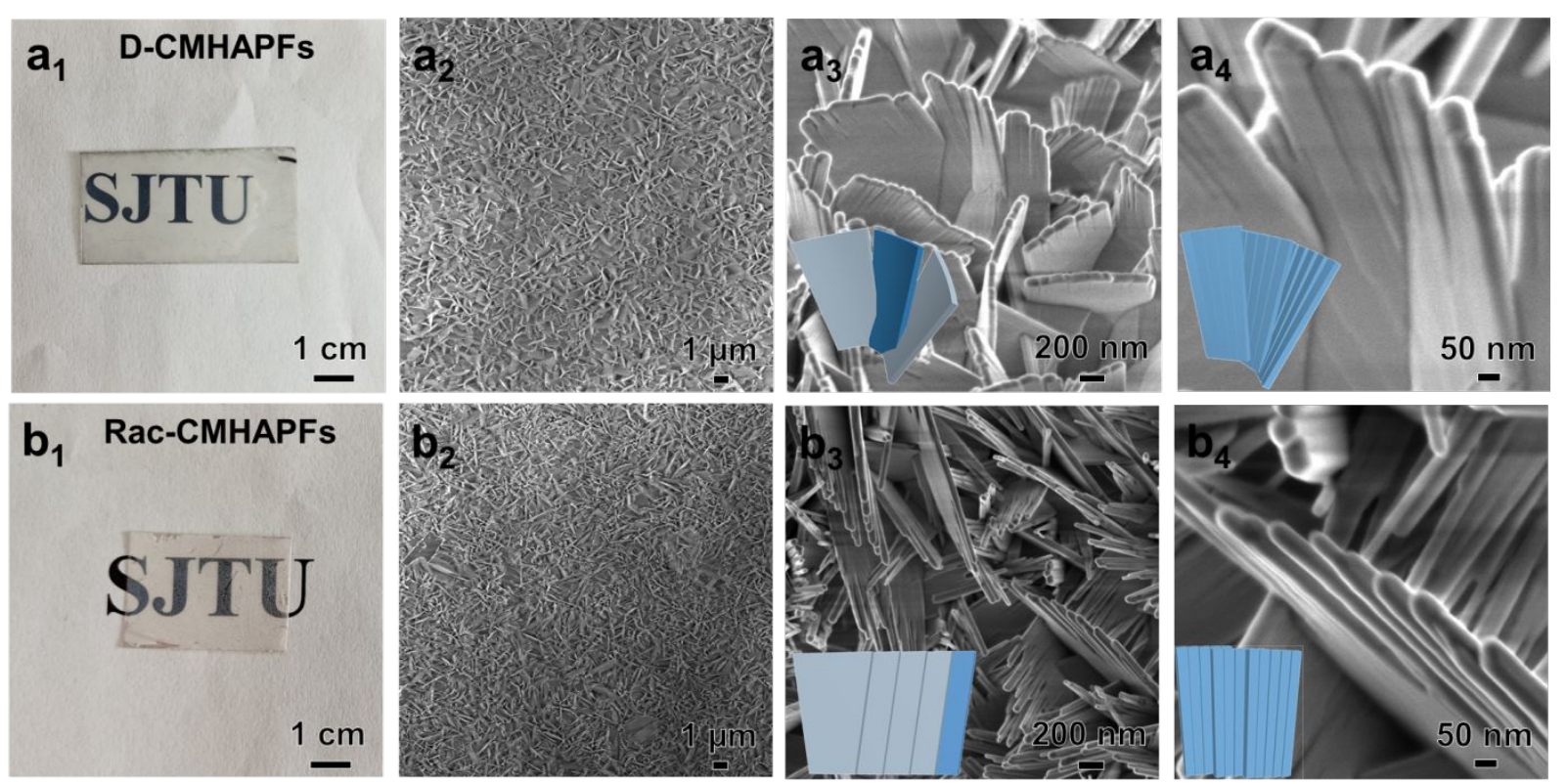

Fig. S2 | Morphologies and structures of the CMHAPFs. a, b, SEM images at varying magnifications and scheme of dense D- and Rac-CMHAPFs, which synthesised with D- and Rac-TTA at $180{ }^{\circ} \mathrm{C}$ for $24 \mathrm{~h}$, respectively. The synthetic molar composition was 0.625 TTA: 1.25 $\mathrm{Ca}\left(\mathrm{NO}_{3}\right)_{2} \bullet 4 \mathrm{H}_{2} \mathrm{O}: 0.75 \mathrm{NH}_{4} \mathrm{H}_{2} \mathrm{PO}_{4}: 1.76 \mathrm{CON}_{2} \mathrm{H}_{4}: 1667 \mathrm{H}_{2} \mathrm{O}$. 


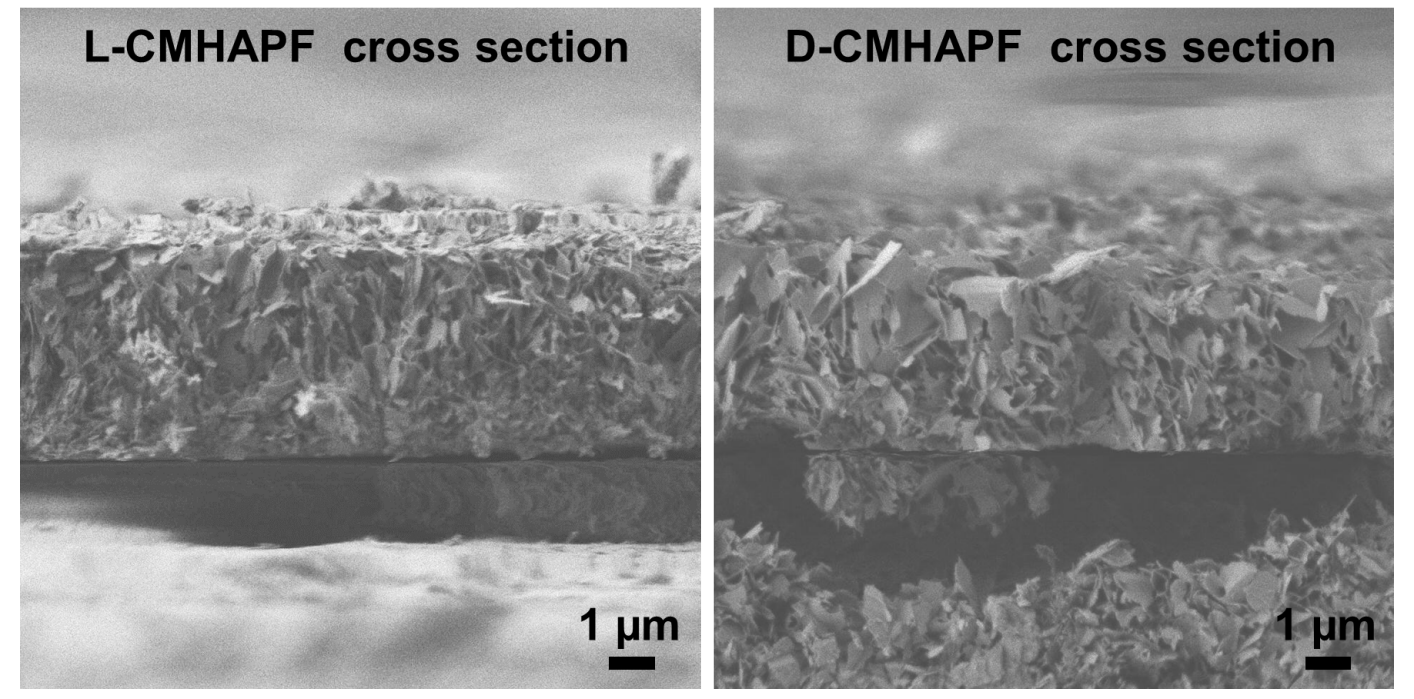

Fig. S3 | Morphologies of the CMHAPFs. Side view of the SEM images of L- and D-CMHAPFs. 


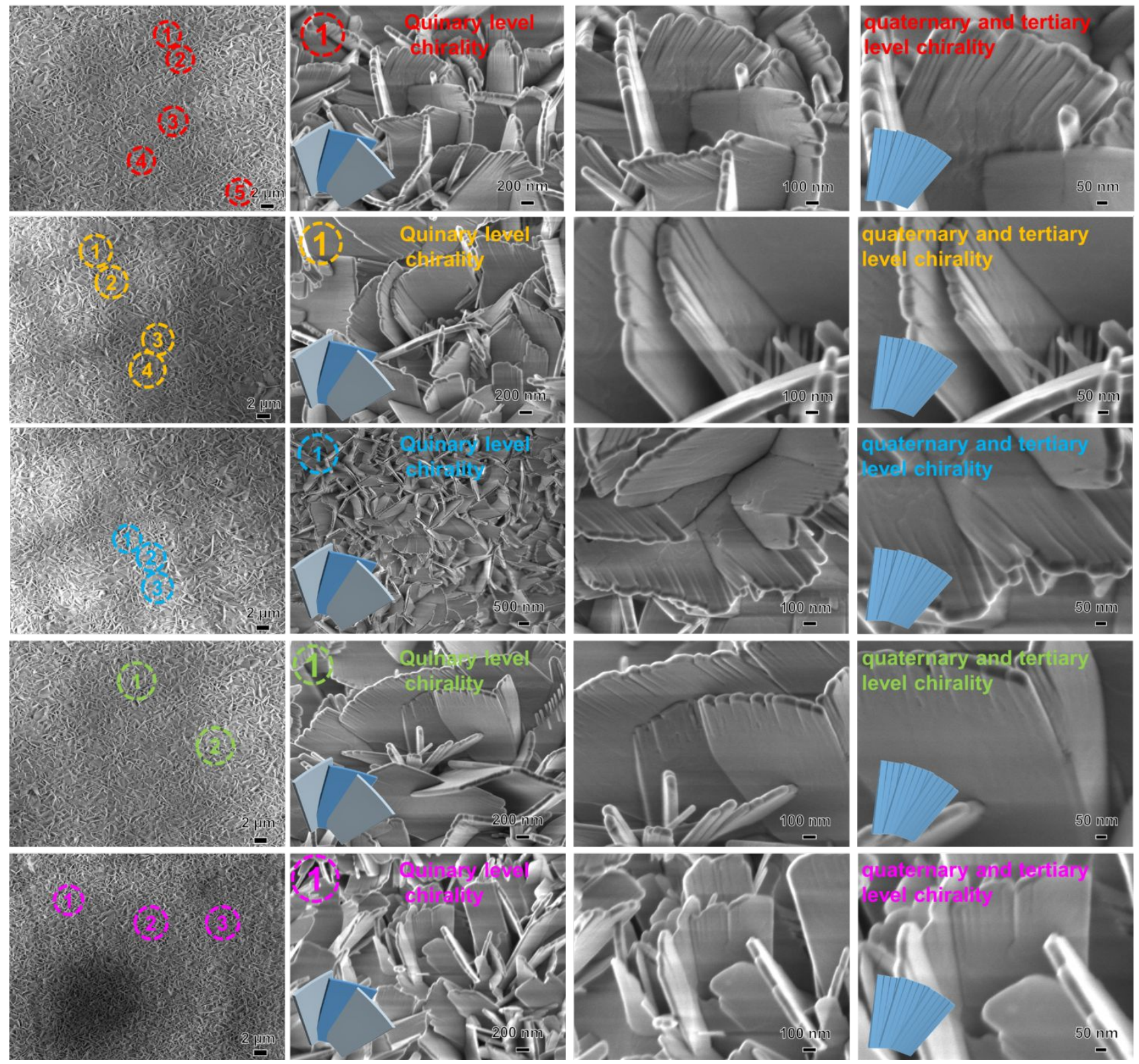

Fig. S4 | SEM images of the repeated L-CMHAPFs. The synthesis molar composition was 0.625 TTA: $1.25 \mathrm{Ca}\left(\mathrm{NO}_{3}\right)_{2} \bullet 4 \mathrm{H}_{2} \mathrm{O}: 0.75 \mathrm{NH}_{4} \mathrm{H}_{2} \mathrm{PO}_{4}: 1.76 \mathrm{CON}_{2} \mathrm{H}_{4}: 1667 \mathrm{H}_{2} \mathrm{O}$. 

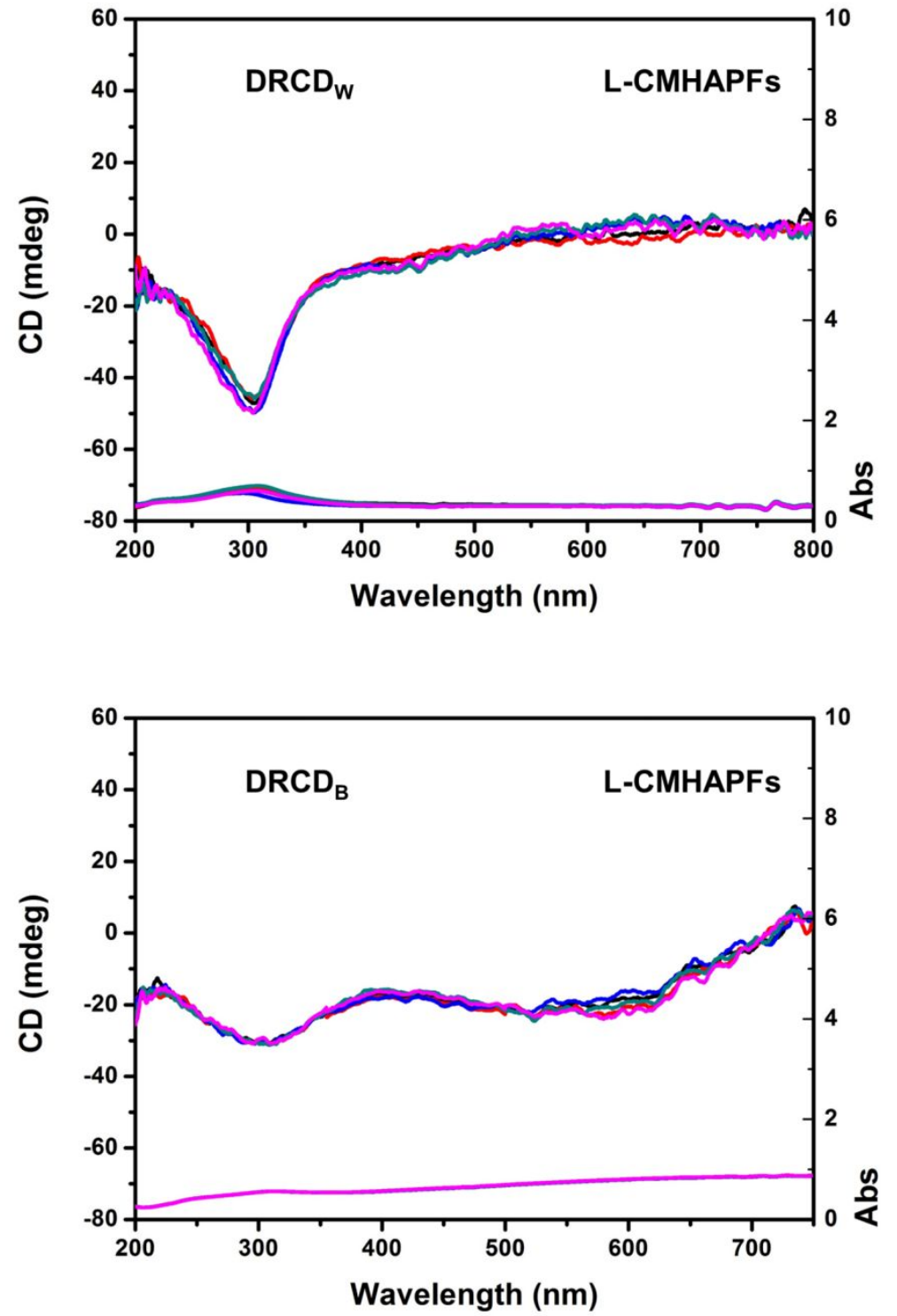

Fig. S5 | Diffuse reflection UV-Vis and CD spectra of the repeated L-CMHAPFs with white $\left(\mathrm{DRCD}_{\mathrm{W}}\right)$ and black $\left(\mathrm{DRCD}_{\mathrm{B}}\right)$ background, respectively. 
a

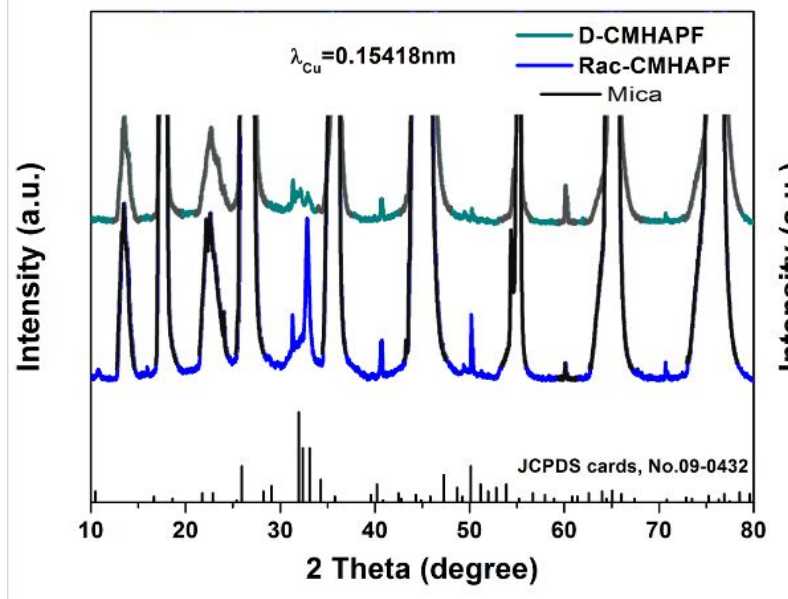

b

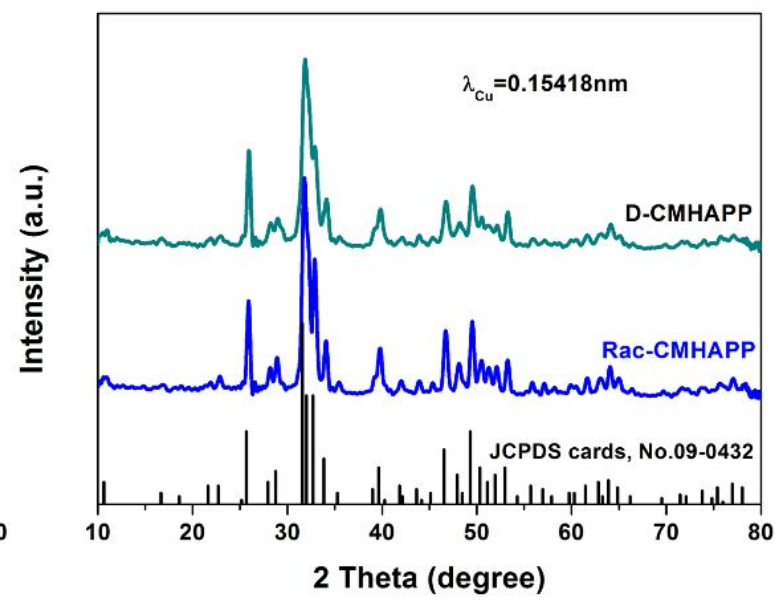

Fig. S6 | Structural feature of the CMHAPPs. The XRD patterns of D-CMHAPF and Rac-CMHAPF (a), D-CMHAPP and Rac-CMHAPP (b) obtained in the same autoclave with corresponding CMHAPFs. 

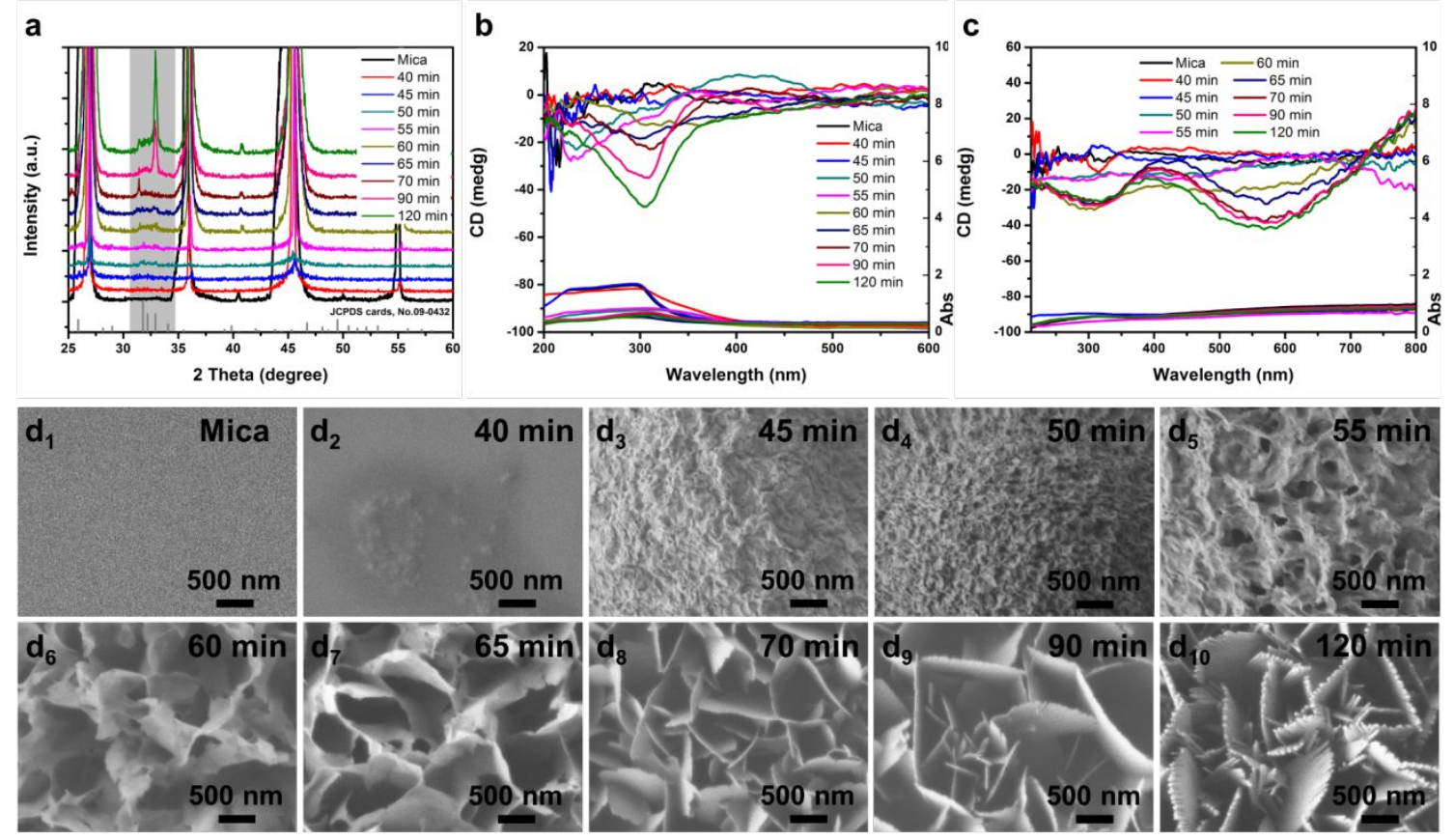

e

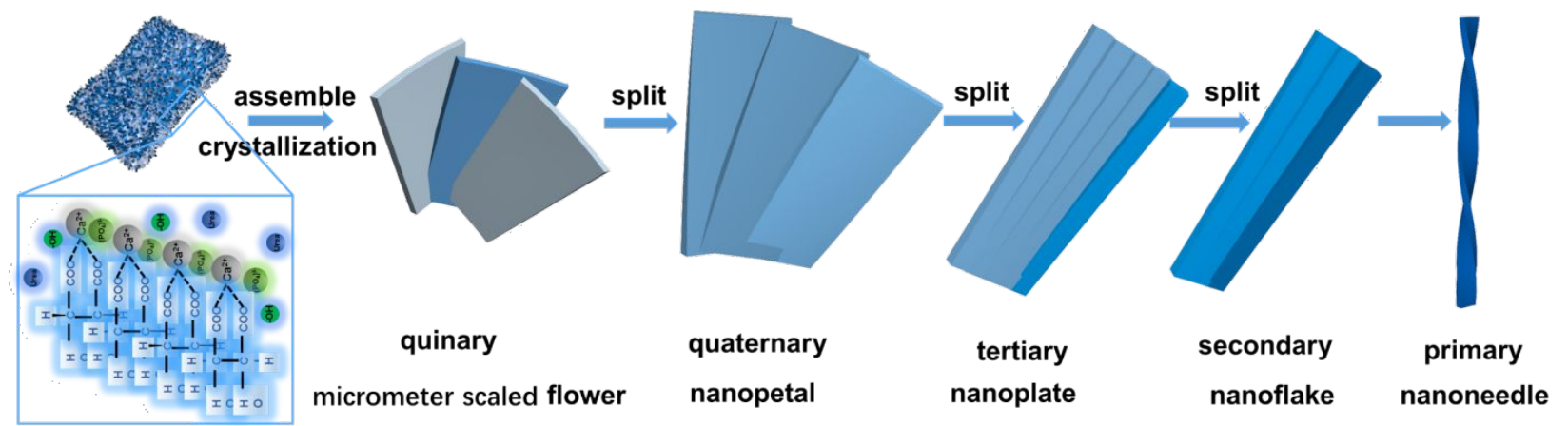

Fig. S7 | The samples synthesized with different reaction times. a, b, c, d, XRD patterns (a); UVVis and CD spectra with white and black background (b and c), respectively; SEM images (d) of LCMHAPFs sampled at different reaction times. e, Illustration the formation mechanism of LCMHAPFs.

Fig. S7 shows the XRD patterns, CD spectra and SEM images of as-prepared L-CMHAPFs at $180{ }^{\circ} \mathrm{C}$ for different reaction times. The HAP nanoparticles firstly seeded on the substrate (40 min), and then growth into amorphous nanosheets with the formation of primary frame (45-55 min), and the hydroxyapatite sheets were further crystallized and assembled into quinary spiral flower like structure (55-70 min). With further increasing the reaction time, the quaternary nanopetals cannot be kept as a whole single crystal over a large distant due to the distortion of the crystal lattice, which split into several tertiary nanoplates, and further the nanoplate split into several secondary nanoflakes and primary naneneedles (70-120 min). During the crystallization process, the XRD intensity and the CD signal gradually enhanced.

As shown in Fig. S7e, the chiral primary structure with left-handed distorted crystal lattice would 
be induced by the L-TTA, in which the carboxyl groups chelated well with the calcium ions. After mixing with phosphate ions and urea under heating, the phosphate ions displace tartrate ion to form calcium phosphate precipitation. During the reaction, the chiral arrangement of TTA molecules would induce the oriented malposed growing of nanocrystals, thus leading to the formation of primary chiral nanoneedles. In another word, the primary chirality of nanoneedles is copied from organic molecules, resulting in the consistence of the primary chirality in the L-CMHAPFs and the chirality of L-TTA assemblies. The secondary nanoflakes, tertiary nanoplates and quaternary nanopetals chirality induced by the continuous twisting of the crystal lattice. The twisted nanopetal grown by nanoneedles epitaxy cannot be kept as a whole single crystal over a large distant due to the distortion of the crystal lattice, which split into several nanoplates, further splitting into nanoflakes. The quinary micrometer scaled 'flower' chirality induced by the left-handed arrangement of left-handed twisted nanopetals. 

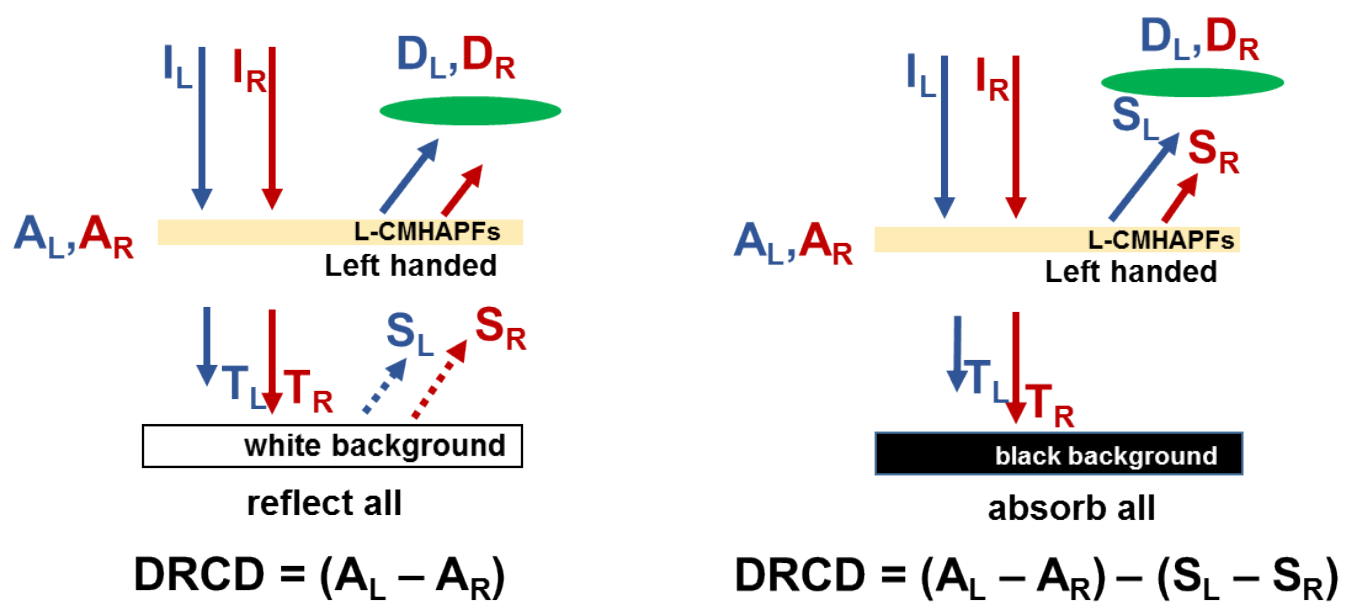

Fig. S8 | Detecting mechanism of DRCD.

The DRUV-vis and DRCD spectra of chiral medium with white background only included the electron transition AOA, CD $=A_{L}-A_{R}$. The DRUV-vis and DRCD spectra of chiral medium with black background included both the electron transition AOA and SOA at the UV-vis range, $C D=\left(A_{L}\right.$ $\left.-A_{R}\right)-\left(S_{L}-S_{R}\right)$. The SOA occurs at integral multiple wavelengths based on $m \lambda=P n_{\text {avg }}$, where $\mathrm{m}$ is integer, $n_{\text {avg }}$ is the average refractive index and $P$ is the pitch length of the chiral medium. The lefthanded structures prefer to absorb right-handed circularly polarized light $\left(A_{L}-A_{R}<0\right)$ revealing negative signals, reflecting left-handed circularly polarized light $\left(\mathrm{S}_{\mathrm{L}}-\mathrm{S}_{\mathrm{R}}>0\right)$ revealing positive signals in transmitted CD while negative signals in DRCD, and vise-versa for the right-handed structure. 

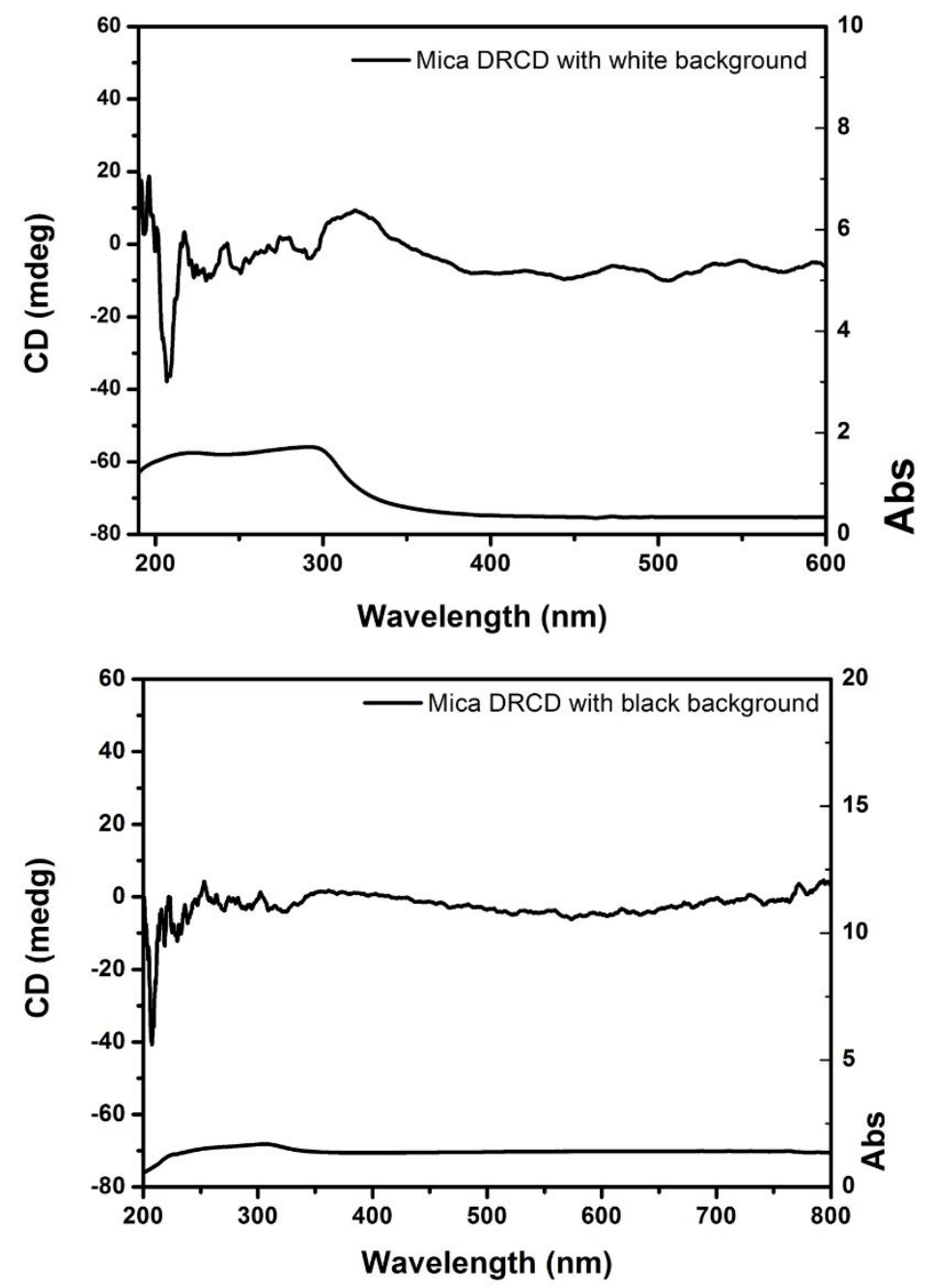

Fig. S9 | OAs of Mica. The diffuse reflectance UV-vis and DRCD spectra of the mica substrates with different background, indicating a weak CD signal expressed in mica substrates. 


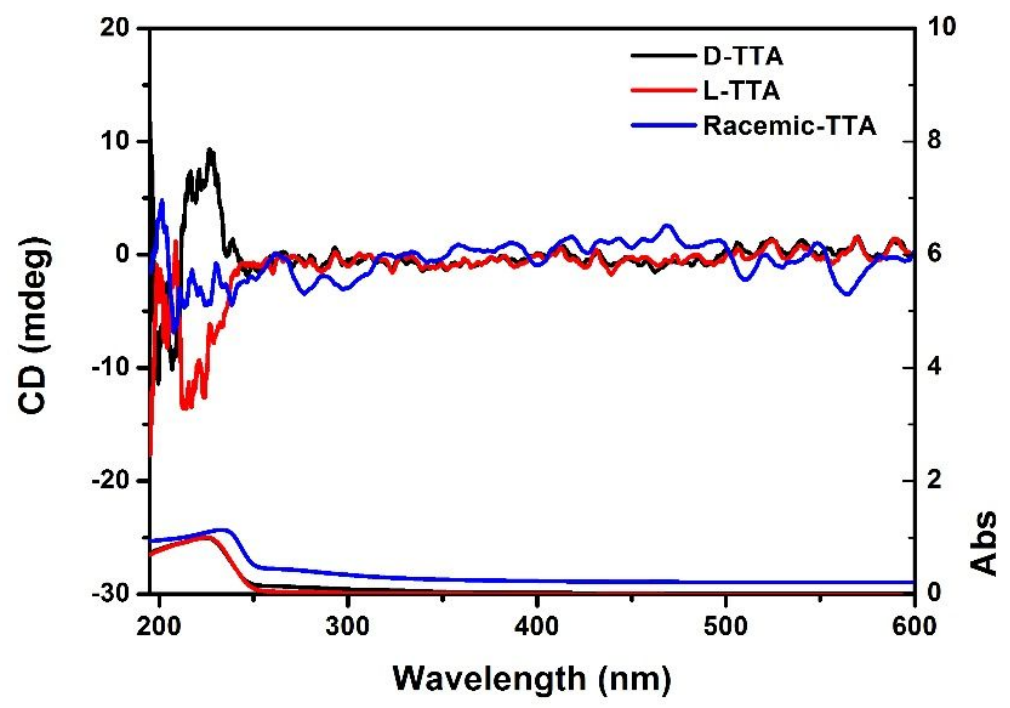

Fig. S10 | OAs of chiral symmetry breaking agents. DRUV-vis absorption and DRCD spectra of D-, L-, Racemic-TTA. 

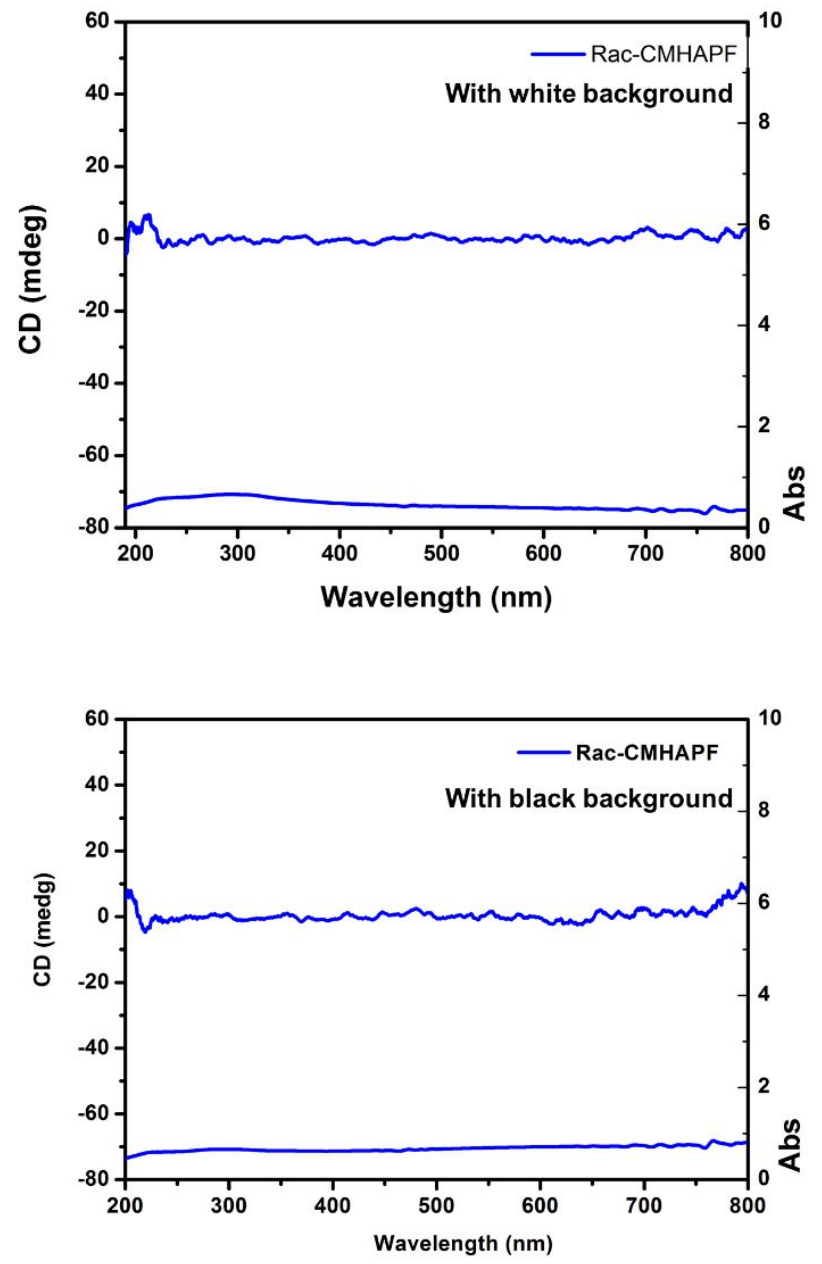

Fig. S11 | OAs of the Rac-CMHAPFs. DRUV-vis absorption and DRCD spectra of the R-CMHAPF measured with a white and black backboard. 


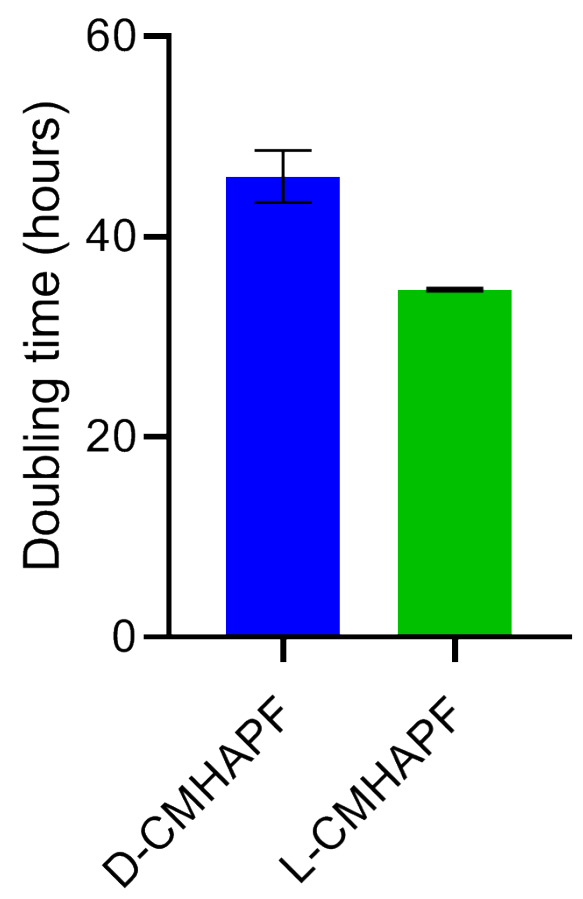

Fig. S12 | Ad-MSC cell proliferation. The doubling time of Ad-MSCs on L-CMHAPFs and DCMHAPFs. 

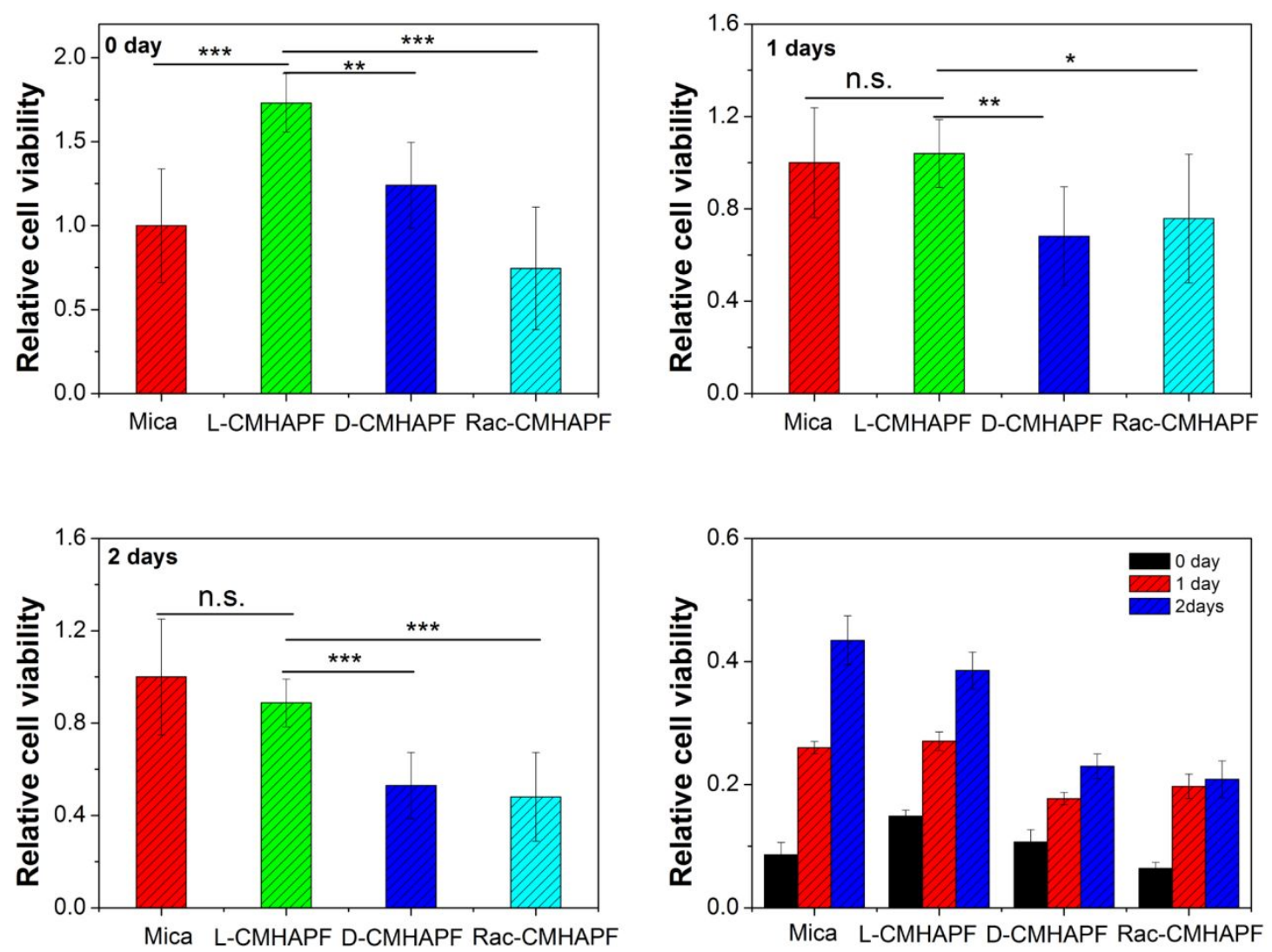

Fig. S13 | Characteristics of Ad-MSCs grown on different substrates. Relative cell viability of AdMSCs cultured on varies substrates for different periods of time. 


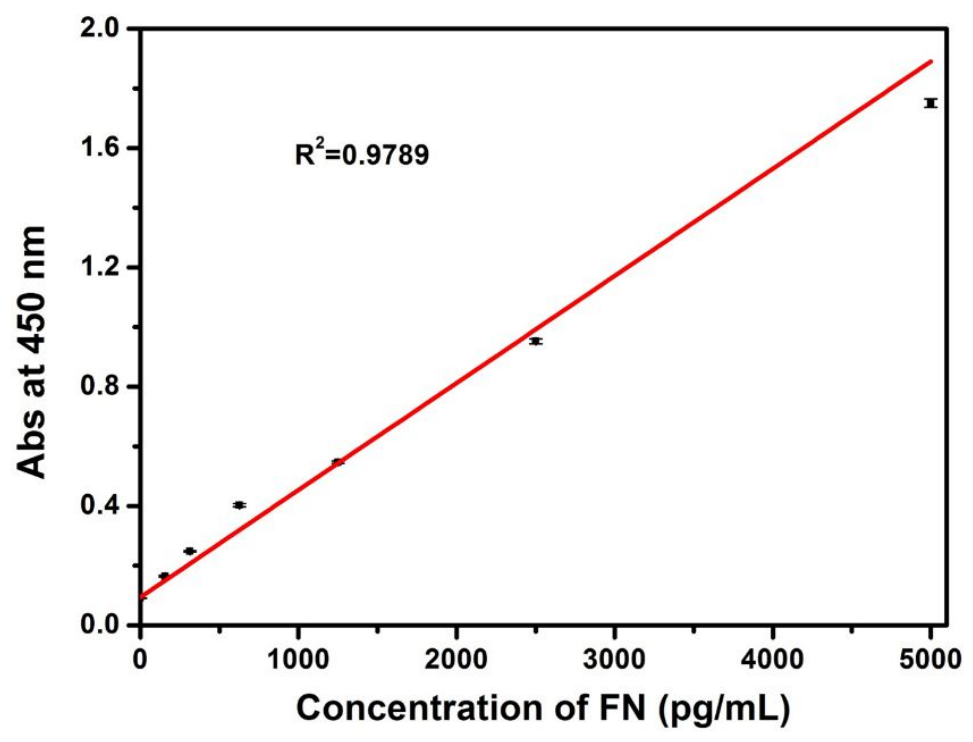

Fig. S14 | FN adsorption standard curve. A plot of Abs at $450 \mathrm{~nm}$ as a function of FN concentrations. The error bars correspond to the standard error of the mean $(n=5)$. 


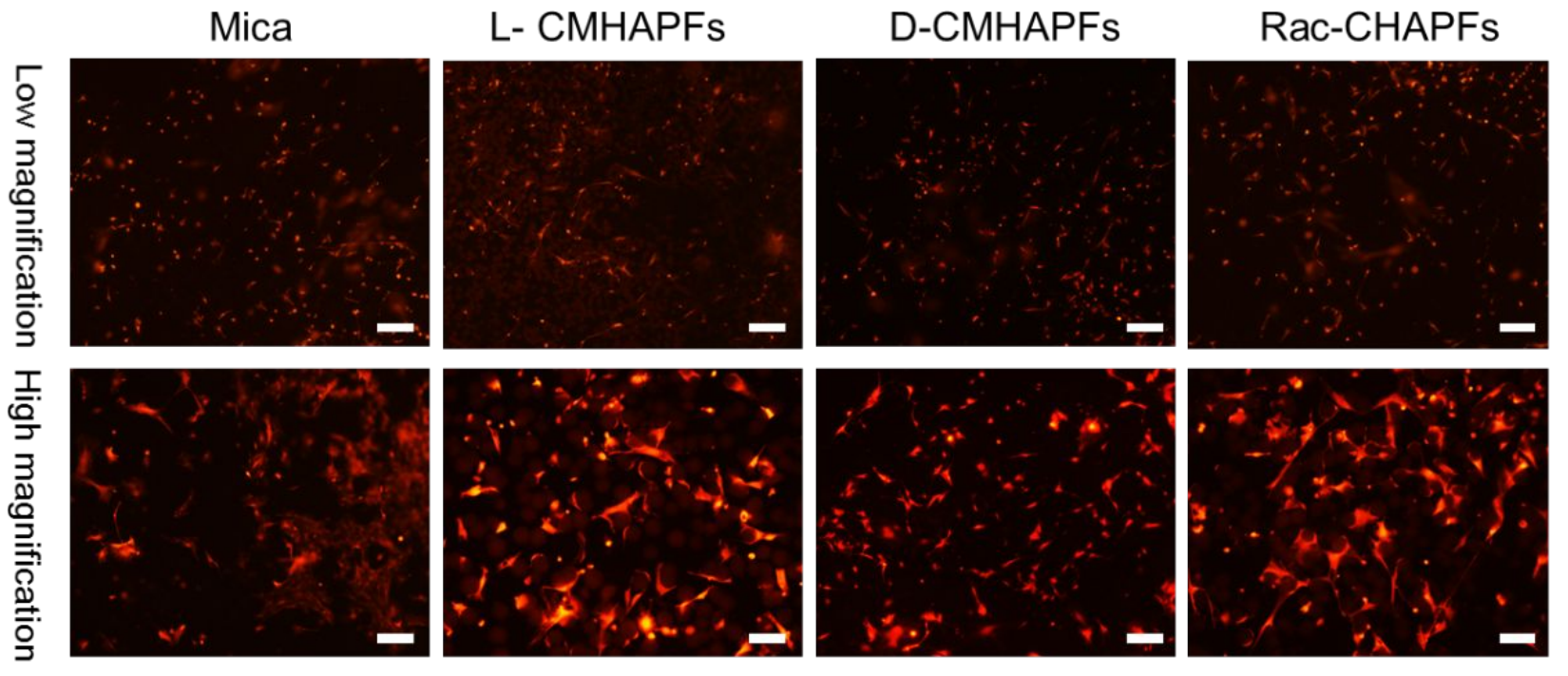

Fig. S15 | Characteristics of BM-MSC cells grown on different substrates. Confocal images of BM-MSC cells derived from the mice where BM-MSCs are genetical labelled type tomato grown on different substrates for $24 \mathrm{~h}$. The scale bars of high-magnification images are $25 \mu \mathrm{m}$, and scale bars of low-magnification images are $75 \mu \mathrm{m}$. 

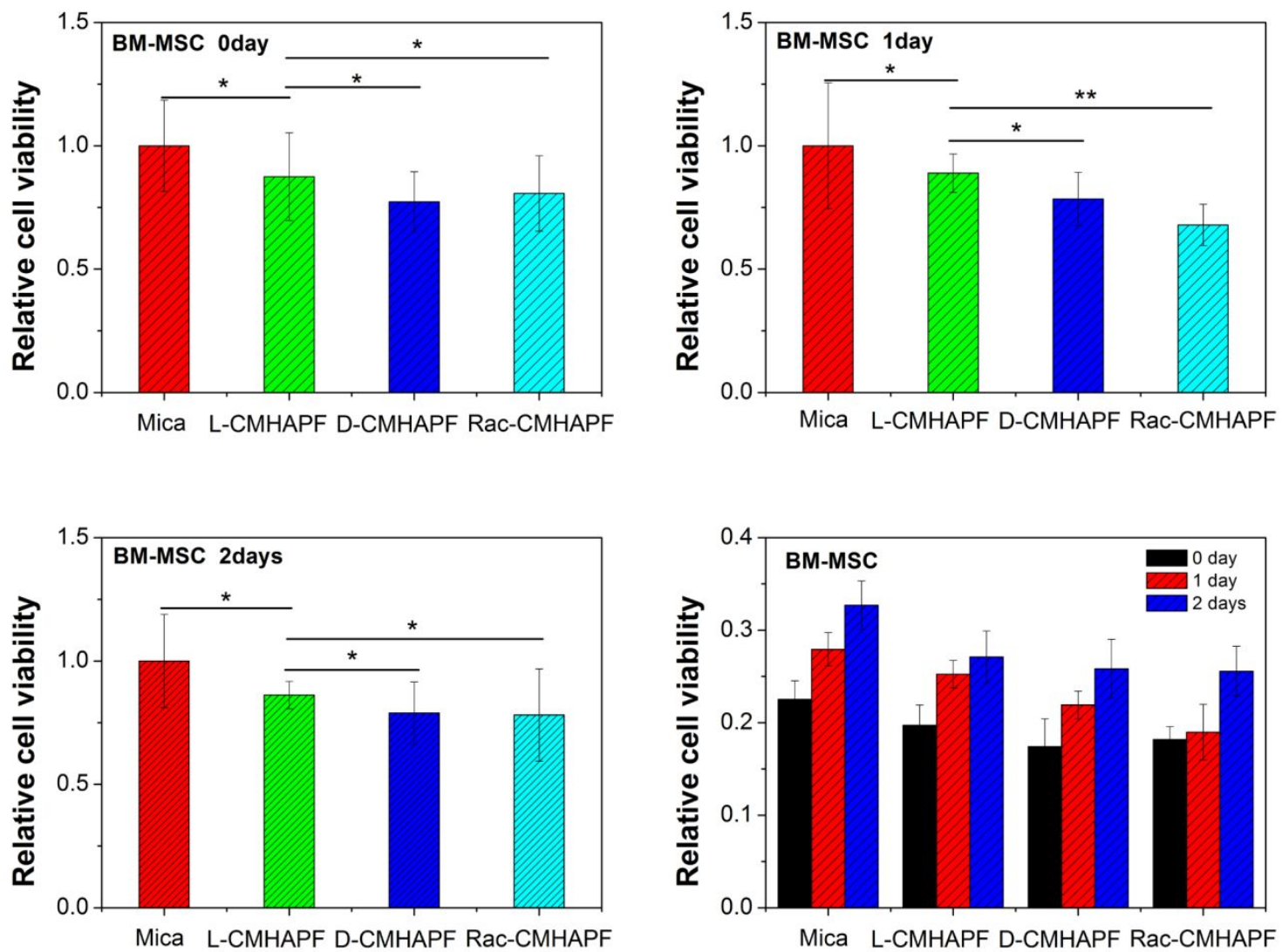

Fig. S16 | Characteristics of BM-MSC cells grown on different substrates. Relative cell viability of BM-MSC cells cultured on varies substrates for different periods of time. 


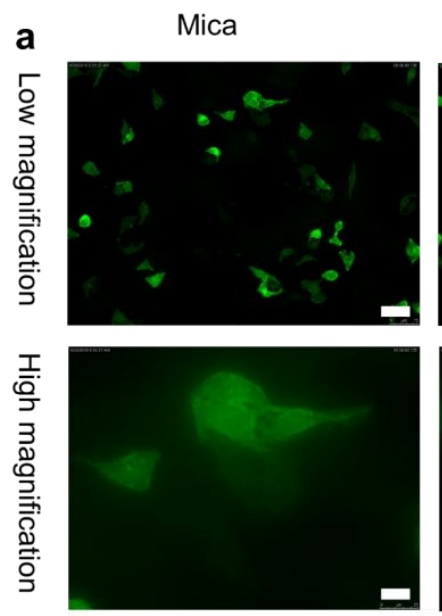

L-CMHAPF
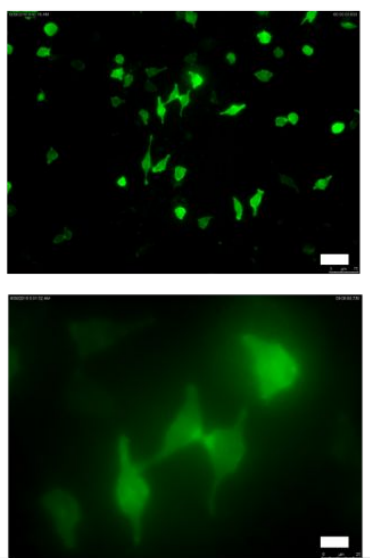

b

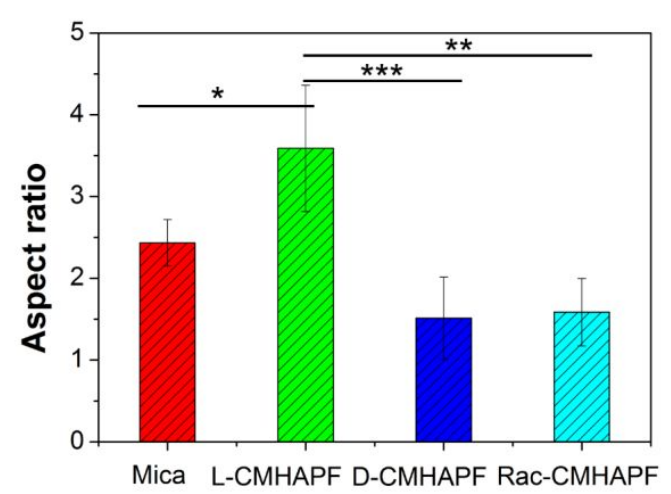

D-CMHAPF
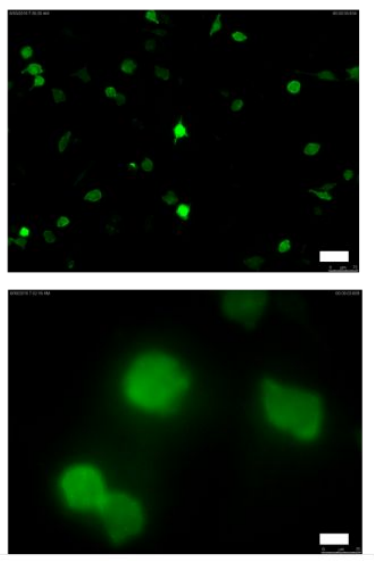

c

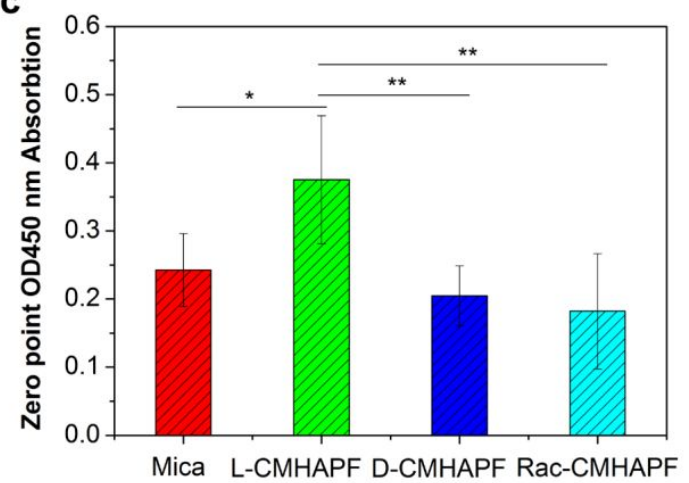

Fig. S17 | Characteristics of MCF-7 cells grown on different chirality substrates. a, Confocal images of MCF-7 cell grown on different substrates (green, cytoplasm) for $4 \mathrm{~h}$. The scale bars of highmagnification images are $25 \mu \mathrm{m}$, and scale bars of low-magnification images are $75 \mu \mathrm{m} . \mathrm{b}$, aspect ratio of MCF-7 cells cultured on different substrates, the error bars correspond to the standard error of the mean $(n=6)$. c, Relative cell viability of MCF-7 cells cultured on varies substrates for 24 hours, the error bars correspond to the standard error of the mean $(n=6)$. "***" refer to a significant difference between the indicated groups: D/L-CMHAPFs and L/R-CMHAPFs (with $p<0.01$ ), LCMHAPFs/mica $(\mathrm{p}<0.05)$. 

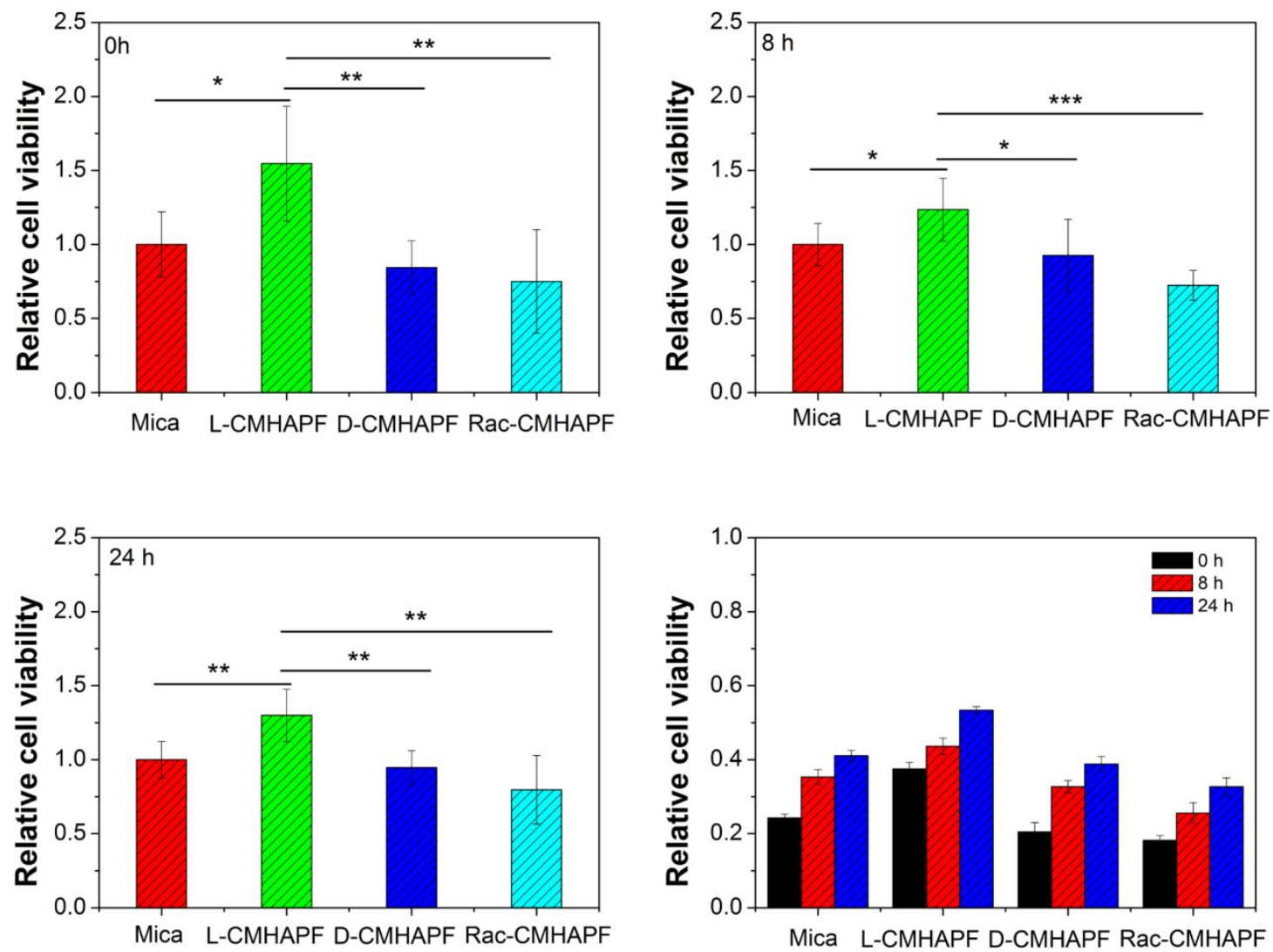

Fig. S18| Characteristics of MCF-7 cells grown on different substrates. Relative cell viability and relative cell densities of MCF-7 cells cultured on varies substrates for different periods of time. The error bars correspond to the standard error of the mean $(n=6) .(* p<0.05, * * p<0.01, * * * p<0.001$, and $* * * * p<0.0001)$. 


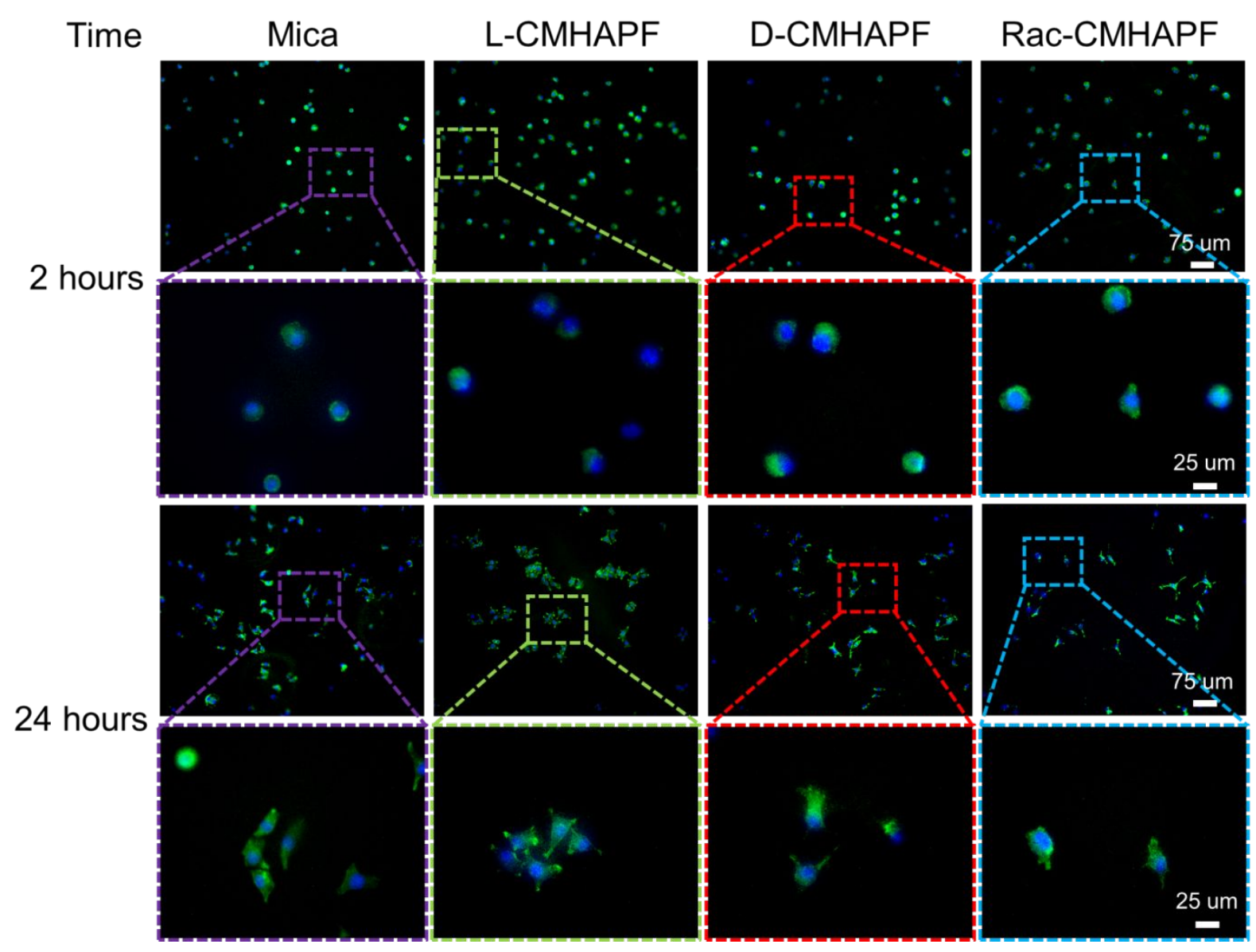

Fig. S19 | Characteristics of L929 cells grown on different substrates. Confocal bright field images of L929 cells grown on different substrates for different periods of time. The scale bars of highmagnification images are $25 \mu \mathrm{m}$, and scale bars of low-magnification images are $75 \mu \mathrm{m}$. 


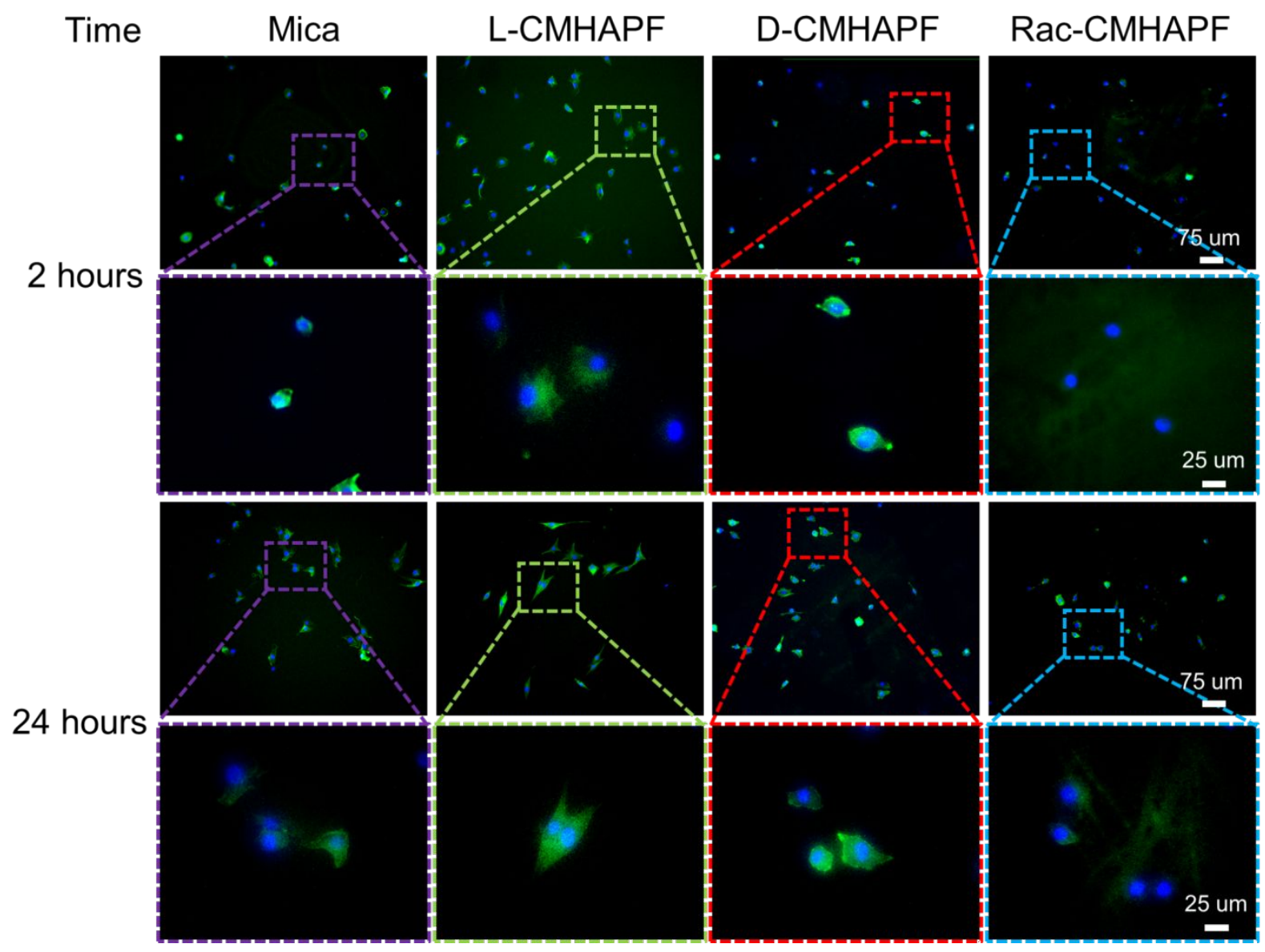

Fig. S20 | Characteristics of 3 T3 cells grown on different substrates. Confocal bright field images of $3 \mathrm{~T} 3$ cells grown on different substrates for different times. The scale bars of high-magnification images are $25 \mu \mathrm{m}$, and scale bars of low-magnification images are $75 \mu \mathrm{m}$.

\section{Movies}

Movie S1. The structure of 3D tomography reconstruction of Ad-MSCs on Mica substrates.

Movie S2. The structure of 3D tomography reconstruction of Ad-MSCs on L-CMHAPFs substrates.

Movie S3. The structure of 3D tomography reconstruction of Ad-MSCs on D-CMHAPFs substrates.

Movie S4. The structure of 3D tomography reconstruction of Ad-MSCs on Rac-CMHAPFs substrates. 